
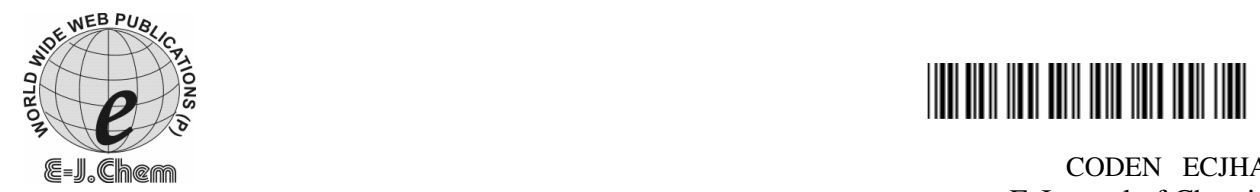

CODEN ECJHAO

E-Journal of Chemistry

http://www.e-journals.net

Vol. 3, No.2, pp 103-109, May 2006

\title{
Synthesis of Novel Azetidinone and Thiazolidinones Derivatives and Evaluation of Their Antimicrobial Efficacy
}

\author{
K.H.PATEL and A.G.MEHTA* \\ Department of Chemistry, \\ P.T.Science College, Surat, Gujarat, India
}

Received 26 February 2006; Accepted 14 April 2006

\begin{abstract}
Amino-4-(2-naphthalenyl) thiazole (1) was prepared from 2acetylnapthalene. This amine on facile condensation with aromatic aldehydes afford Schiff Base/anils/azomethines(2a-h). These anils on cyclocondensation reaction with chloro acetyl chloride and thio glycolic acid (i.e. mercapto acetic acid) yields 2-azetidinones and 4-thiazolidinones respectively. The prepared compounds have been screened on some stains of bacteria.
\end{abstract}

Key words: 2-Azetidinones, 4-thiazolidinones, cyclo-condenction reaction, spectral studies antibacterial action.

\section{Introduction}

Thiazoles are one of the most intensively investigated classes of aromatic five membered hetrocycles. Thiazole derivatives find new a variety of applications ranging from bacteriostatics, antibiotics, CNS regulants of high selling diureties ${ }^{1-5}$. All these facts were driving force to develop novel thiozole derivatives with wide structural varation ${ }^{6}$. Thus thiazole derivatives playes pivotal role in medicinal chemistry.

As part of interest in hetrocycles that have been explored for developing pharmaceutically important molecules, 4-thiazolidinones ${ }^{7-9}$ and 2 -azetidinones ${ }^{10-13}$ have played an important role in medicinal chemistry. Moreover they have been studied extensively because of their ready accessibility, diverse chemical reactivity and brode spectrum of biological activity. The area in which the transformation of 2-amino-4-(2naphthalenyl) thiazole into azetidinones and thiazolidinones has not reported so far. Hence it was thought interesting to study such type of moieties shown in scheme-1. 


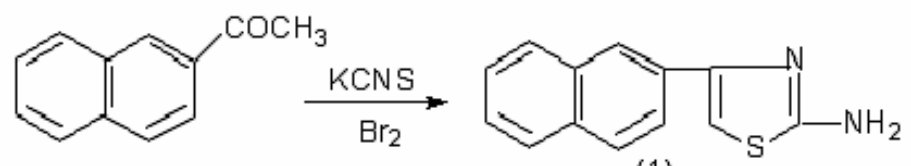

(1)

2-Acetyl naphthalene

2-amino-4-(2-naphthalenyl) thiazole

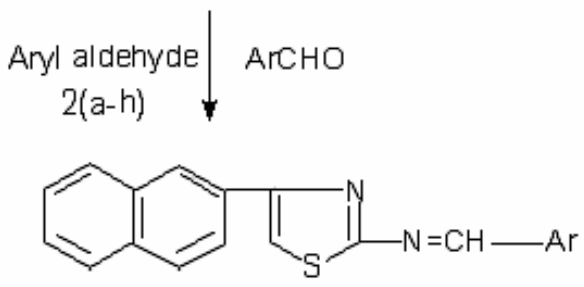

Schiff Base

$(3 a-h)$

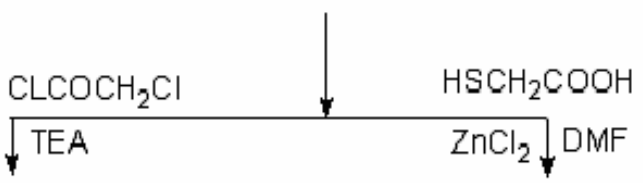<smiles>O=C1N(c2nc(-c3ccc4ccccc4c3)cs2)C(Cl)C1(Br)Br</smiles>

$4 a-h$<smiles>O=C1CSC([Al])N1c1nc(-c2ccc3ccccc3c2)cs1</smiles>

$5 a-h$

Scheme-1

Where Ar: (a) Phenyl, (b) 4-Methoxy Phenyl (c) 4-Hydroxy Phenyl (d) 2-Hydroxy Phenyl (e) 4-Methyl Phenyl (f) 3,4-Methylenedioxy Phenyl (g) 4-Hydroxy3-Methoxy Phenyl (h) 3,4-Diethoxy Phenyl

\section{Experimental}

\section{Materials}

2-Amino-4-(2-naphthalenyl) thiazole was prepared according to method reported ${ }^{14}$. The aromatic benzadelydes (2a-h) viz; a: benzaldehyde, b: 4-methoxy benzaldehyde, c: 4-hydroxy benzaldehyde, d: 2- hydroxybenzaldehyde, e: 4- methyl benzaldehyde, f: 4-bramobenzaldelhyde, g: 3,4-ethylenedioxy benzaldehyde, h: 4-hydroxy-3-methoxy benzaldehyde and i: 3,4-dimethoxy benzaldehyde were obtained from local dealer. All other chemicals used where of laboratory grade.

\section{Measurements}

Melting points were determined in open capillary tubes and were uncorrected. The IR spectra were recorded in KBR pellets on a nicolet 760D spectrophotometer and 1H NMR spectra in $\mathrm{CDCI}_{3}$ on Perkin Elmar NMR spectrometer using TMS as an internal standard. 
Antimicrobial activity of all the compounds were studied against Gram positive bacteria (Bacillus Subtillies and staphycoccus aureus) and Gram negative bacteria (E.Coli and salmonella typhi) at a concentration of $50 \mu \mathrm{g} / \mathrm{ml}$ by agar cup method ${ }^{15}$.Methanol system was used as control in the method. Under similar conditions using penicillin and sulfanilamide as a standard comparison carried at control experiment. The area of inhibition of zone is measured in percentage

\section{Preparation of Schiff base (3a-h)}

A mixture of equiolar amount (0.01) of 2-amino-4-(2-naphthalenyl) thiazole (1) benzaldehyde derivative (2a-h) in ethanol $(40 \mathrm{ml})$ and piperidine $(0.3 \mathrm{ml})$ was refluxed for 5 hrs on water bath. The reaction mixture was concentrated, cooled and poured in water; the solid obtained was filtered and recrystallised from ethanol to give Schiff base (3a-h). It was obtained in $60-65 \%$ yield.

\section{Preparation of 2-Azetidionones (4a-h)}

A mixture of Schiff base (3a-h) $(0.002 \mathrm{mmol})$ and triethyl amine [TEA] $(0.004 \mathrm{mmol})$ was dissolved in 1,4-dioxane $(50 \mathrm{ml})$, cooled and stirred. To this well-stirred cooled solution chloro acetyl chloride $(0.004 \mathrm{mmol})$ was added drop wise with in a period of $20 \mathrm{~min}$. The reaction mixture was then stirred for an additional $3 \mathrm{hrs}$ and left at room temperature for 48 hrs. The resultant mixture was concentrated, cooled, poured in to ice cold water, filter and then dired. The product thus obtained was purified by column chromatography over silica gel using 30\% ethyl acetate: $70 \%$ benzene as an eluent. Recrystallization from either / n-Hexane gave 2-azetidinones (4a-h), which were obtained in 55-60\% yield.

\section{Preparation of 2-Thiazolidinones (5a-h)}

A mixture of Schiff base (3a-h) $(0.01 \mathrm{mmol})$ in THF $(30 \mathrm{ml})$ and mercapto acetic acid $(0.01$ mmol) with a pinch of anhydrous $\mathrm{ZnCl}_{2}$ was then refluxed to at a residue, which was dissolved in 1,4-dioxane passed through a column of silica gel using benzene : chloroform $(8: 2, \mathrm{v} / \mathrm{v})$ mixture as an eluent. The eluent was concentrated and the product recrystallized 4-thiazolidinoines (5a-h) from ethanol: 1,4-dioxane (1:1) mixture 50-60\% yield.The analytical data of all the compounds $\mathbf{4 a}-\mathbf{i}$ and $\mathbf{5 a - i}$ are furnished in Tables 1 and 2.

\section{Results and Discussion}

The 2-Amino-4-(2-naphthalenyl)thiazole(2) was dissolved in ethanol and was reacted with aeromatic aldehyde in the presence of piperidine to yield Schiff bases (3a-h). This Schiff bases (3a-h) were then characterized by the elemental analysis, IR spectral studies and NMR spectral studies. The IR spectra of Schiff bases show the prominent band at $1630 \mathrm{~cm}^{-1}$ for the azomethine group ${ }^{16,17}$.

These Schiff bases on cyclo-condensation reaction with chloro acetyl chloride afford 2azetidinone (4a-h) and with thio-glycolic acid afford 4-thiazolidinone (5a-h) respectively. The structures of both these compounds (4a-h) and (5a-h), respectively, have been confirmed by elemental analysis, IR spectral studies, and NMR spectral studies. These compounds shows the band at $1690 \mathrm{~cm}^{-1}$ for cyclic $>\mathrm{C}=\mathrm{O}$ group ${ }^{16,17}$. All the compounds show the NMR signals for different kinds of protons at their respective positions. The data are shown in Tables 1 and 2 . The $\mathrm{C}, \mathrm{H}, \mathrm{N}, \mathrm{S}$ analysis of all the compounds of the series are presented in Table 1 and 2. The values are consistent with their predicted structure (Scheme 1). 
Table 1 Analytical and spectral Data of Commands 4a-h

\begin{tabular}{|c|c|c|c|c|c|c|c|c|c|c|c|c|c|}
\hline \multirow{3}{*}{ Compd } & \multirow{3}{*}{$\begin{array}{l}\text { Molecular } \\
\text { formula }\end{array}$} & \multirow{3}{*}{$\begin{array}{l}\text { Mol. } \\
\text { Wt }\end{array}$} & \multirow{3}{*}{$\begin{array}{c}\text { Yield } \\
(\%)\end{array}$} & \multirow{3}{*}{$\begin{array}{l}\text { M.P. } \\
\left({ }^{\circ} \mathrm{C}\right)\end{array}$} & \multicolumn{8}{|c|}{$\%$ Analysis } & \multirow{3}{*}{ PMR (ס PPM) } \\
\hline & & & & & \multicolumn{2}{|c|}{$\% \mathrm{C}$} & \multicolumn{2}{|c|}{$\% \mathrm{H}$} & \multicolumn{2}{|c|}{$\% \mathrm{~N}$} & \multicolumn{2}{|c|}{$\% \mathrm{~S}$} & \\
\hline & & & & & Cald & Found & Cald & Found & Cald & Found & Cald & Found & \\
\hline $4 a$ & $\mathrm{C}_{12} \mathrm{H}_{15} \mathrm{ClN}_{2} \mathrm{OS}$ & 390.5 & 65 & $182-3$ & 67.6 & 67.5 & 3.84 & 3.9 & 7.17 & 71 & 8.19 & 8.1 & $\begin{array}{l}9-21 \mathrm{H} \mathrm{d} \mathrm{C} \mathrm{C}_{3} \mathrm{H} \text { 9-2-7-8 }(12 \\
\mathrm{H} \text { mtd aromatic } \mathrm{C}_{4} \mathrm{H}\end{array}$ \\
\hline $4 b$ & $\mathrm{C}_{23} \mathrm{H}_{17} \mathrm{ClN}_{2} \mathrm{O}_{2} \mathrm{~S}$ & 420.5 & 61 & $178-9$ & 65.5 & 65.4 & 4.04 & 3.9 & 6.65 & 66 & 7.60 & 7.5 & $\begin{array}{l}\text { 8-2-7.8 }(11 \mathrm{H} \mathrm{mtd} \text { aromatic } \\
\& \mathrm{C}_{4} \mathrm{H}, 9.2\left(\mathrm{H} \mathrm{d} . \mathrm{C}_{3} \mathrm{H}\right) \\
2.1\left(3 \mathrm{H}_{3} \mathrm{CH}_{3}\right)\end{array}$ \\
\hline $4 c$ & $\mathrm{C}_{22} \mathrm{H}_{15} \mathrm{ClN}_{2} \mathrm{O}_{2} \mathrm{~S}$ & 406.5 & 55 & $170-1$ & 64.9 & 67.8 & 3.69 & 3.6 & 6.88 & 68 & 7.87 & 7.8 & $\begin{array}{l}\text { 8-2-9.8 }(11 \mathrm{H} \mathrm{mtd} \text { aromatic } \\
\& \mathrm{C}_{4} \mathrm{H}, 9.2\left(\mathrm{H} \mathrm{d} . \mathrm{C}_{3} \mathrm{H}\right) \\
3.9(\mathrm{H} \mathrm{S} \mathrm{OH})\end{array}$ \\
\hline $4 d$ & $\mathrm{C}_{22} \mathrm{H}_{15} \mathrm{ClN}_{2} \mathrm{O}_{2} \mathrm{~S}$ & 406.5 & 60 & $190-1$ & 64.9 & 64.8 & 3.69 & 3.6 & 6.88 & 68 & 7.87 & 7.8 & $\begin{array}{l}\text { 8-2-9.8 }(11 \mathrm{H} \mathrm{mtd} \text { aromatic } \\
\& \mathrm{C}_{4} \mathrm{H}, 9.2\left(\mathrm{H} \mathrm{d} . \mathrm{C}_{3} \mathrm{H}\right) \\
3.9(\mathrm{H} \mathrm{S} \mathrm{OH})\end{array}$ \\
\hline $4 e$ & $\mathrm{C}_{23} \mathrm{H}_{15} \mathrm{ClN}_{2} \mathrm{O}_{2} \mathrm{~S}$ & 406.5 & 65 & $103-4$ & 68.2 & 68.1 & 4.20 & 4.1 & 6.92 & 68 & 7.91 & 7.8 & $\begin{array}{l}\text { 8-2-9.8 }(11 \mathrm{H} \mathrm{mtd} \text { aromatic } \\
\& \mathrm{C}_{4} \mathrm{H}, 9.2\left(\mathrm{H} \mathrm{d} . \mathrm{C}_{3} \mathrm{H}\right) \\
3.9(\mathrm{H} \mathrm{S} \mathrm{OH})\end{array}$ \\
\hline $4 \mathrm{f}$ & $\mathrm{C}_{23} \mathrm{H}_{15} \mathrm{ClN}_{2} \mathrm{O}_{3} \mathrm{~S}$ & 434.5 & 56 & $151-2$ & 63.5 & 63.4 & 3.45 & 4.3 & 6.44 & 64 & 7.36 & 7.2 & $\begin{array}{l}\text { 8-2-7.8 }(11 \mathrm{H} \mathrm{mtd} \text { aromatic } \\
\& \mathrm{C}_{4} \mathrm{H}, 9.2\left(\mathrm{H} \mathrm{d} . \mathrm{C}_{3} \mathrm{H}\right) \\
3.9(\mathrm{H} \mathrm{S} \mathrm{OH})\end{array}$ \\
\hline $4 \mathrm{~g}$ & $\mathrm{C}_{23} \mathrm{H}_{17} \mathrm{ClN}_{2} \mathrm{O}_{2} \mathrm{~S}$ & 436.5 & 52 & $188-9$ & 63.2 & 63.1 & 3.89 & 3.8 & 6.41 & 63 & 7.33 & 7.2 & $\begin{array}{l}\text { 8-2-9.8 }(11 \mathrm{H} \mathrm{mtd} \text { aromatic } \\
\& \mathrm{C}_{4} \mathrm{H}, 9.2\left(\mathrm{H} \mathrm{d} . \mathrm{C}_{3} \mathrm{H}\right) \\
3.9(\mathrm{H} \mathrm{S} \mathrm{OH})\end{array}$ \\
\hline $4 \mathrm{~h}$ & $\mathrm{C}_{24} \mathrm{H}_{19} \mathrm{ClN}_{2} \mathrm{O}_{3} \mathrm{~S}$ & 450.5 & 45 & $169-7$ & 63.9 & 63.8 & 4.21 & 4.1 & 6.21 & 61 & 7.10 & 7.0 & $\begin{array}{l}\text { 8-2-9.8 }(11 \mathrm{H} \mathrm{mtd} \text { aromatic } \\
\& \mathrm{C}_{4} \mathrm{H}, 9.2\left(\mathrm{H} \mathrm{d} . \mathrm{C}_{3} \mathrm{H}\right) \\
3.9(\mathrm{H} \mathrm{S} \mathrm{OH})\end{array}$ \\
\hline
\end{tabular}


Table 2 Analytical and spectral Data of Commands Thiazolidinones, 5a-h

\begin{tabular}{|c|c|c|c|c|c|c|c|c|c|c|c|c|c|}
\hline \multirow{3}{*}{ Compd } & \multirow{3}{*}{$\begin{array}{l}\text { Molecular } \\
\text { formula }\end{array}$} & \multirow{3}{*}{$\begin{array}{l}\text { Mol. } \\
\text { Wt }\end{array}$} & \multirow{3}{*}{$\begin{array}{l}\text { Yield } \\
(\%)\end{array}$} & \multirow{3}{*}{$\begin{array}{l}\text { M.P. } \\
\left({ }^{\circ} \mathrm{C}\right)\end{array}$} & \multicolumn{8}{|c|}{$\%$ Analysis } & \multirow{3}{*}{ PMR (8 PPM) } \\
\hline & & & & & \multicolumn{2}{|c|}{$\% \mathrm{C}$} & \multicolumn{2}{|c|}{$\% \mathrm{H}$} & \multicolumn{2}{|c|}{$\% \mathrm{~N}$} & \multicolumn{2}{|c|}{$\% \mathrm{~S}$} & \\
\hline & & & & & Cald & Found & Cald & Found & Cald & Found & Cald & Found & \\
\hline $5 a$ & $\mathrm{C}_{22} \mathrm{H}_{15} \mathrm{~N}_{2} \mathrm{OS}_{2}$ & 387 & 65 & $160-1$ & 68.1 & 8.1 & 3.87 & 3.8 & 7.23 & 7.2 & 16.5 & 16.4 & $\begin{array}{l}4.3\left(\mathrm{~s}, 1 \mathrm{H} \mathrm{C}_{2} \mathrm{H}, \mathrm{C}_{3} \mathrm{H}\right) \\
6.2 .-7-8(12 \mathrm{H} \mathrm{mtd} \text { aromatic } \\
\left.\mathrm{C}_{4} \mathrm{H}\right), 1.25\left(\mathrm{~S}, 2 \mathrm{H}, \mathrm{CH}_{2}\right)\end{array}$ \\
\hline $5 b$ & $\mathrm{C}_{23} \mathrm{H}_{17} \mathrm{~N}_{2} \mathrm{O}_{2} \mathrm{~S}_{2}$ & 417 & 70 & $165-6$ & 66.1 & 66.0 & 4.07 & 4.0 & 6.71 & 6.6 & 15.3 & 15.2 & $\begin{array}{l}8.2-7.8(11 \mathrm{H} \mathrm{mtd} \text { aromatic } \\
\mathrm{C}_{4} \mathrm{H}, 4.6\left(1 \mathrm{H} \mathrm{d} . \mathrm{C}_{3} \mathrm{H}\right), 125 \\
\mathrm{~S}, 1 \mathrm{H}, \mathrm{C}_{2} \mathrm{H}, 3.4(3 \mathrm{H} \mathrm{SOCH})\end{array}$ \\
\hline $5 c$ & $\mathrm{C}_{22} \mathrm{H}_{15} \mathrm{~N}_{2} \mathrm{O}_{2} \mathrm{~S}_{2}$ & 403 & 63 & $175-6$ & 65.5 & 65.4 & 3.72 & 3.6 & 6.94 & 6.8 & 15.8 & 15.38 & $\begin{array}{l}\text { 6.2-7.8 }(11 \mathrm{H} \mathrm{mtd} \text { aromatic } \\
\mathrm{C}_{4} \mathrm{H}, 4.6\left(1 \mathrm{H} \mathrm{d} . \mathrm{C}_{3} \mathrm{H}\right) \\
3.4(3 \mathrm{H} \mathrm{S} \mathrm{OH}\end{array}$ \\
\hline $5 \mathrm{~d}$ & $\mathrm{C}_{22} \mathrm{H}_{15} \mathrm{~N}_{2} \mathrm{O}_{2} \mathrm{~S}_{2}$ & 403 & 71 & $158-9$ & 65.5 & 68.4 & 3.72 & 3.6 & 6.94 & 6.8 & 15.8 & 15.7 & $\begin{array}{l}\text { 6.2-7.8 }(11 \mathrm{H} \mathrm{mtd} \text { aromatic } \\
\mathrm{C}_{4} \mathrm{H}, 4.6\left(1 \mathrm{H} \mathrm{d} . \mathrm{C}_{3} \mathrm{H}\right) \\
3.4(3 \mathrm{H} \mathrm{S} \mathrm{OH}\end{array}$ \\
\hline $5 e$ & $\mathrm{C}_{23} \mathrm{H}_{17} \mathrm{~N}_{2} \mathrm{OS}_{2}$ & 415 & 60 & $183-4$ & 66.5 & 65.4 & 4.09 & 4.0 & 6.74 & 6.6 & 15.4 & 15.3 & $\begin{array}{l}\text { 8.2-7.8 }(11 \mathrm{H} \mathrm{mtd} \text { aromatic } \\
\mathrm{C}_{4} \mathrm{H}, 4.6\left(1 \mathrm{H} \mathrm{d} . \mathrm{C}_{3} \mathrm{H}\right), 125 \\
\mathrm{~S}, 1 \mathrm{H}, \mathrm{C}_{2} \mathrm{H}, 3.4(3 \mathrm{H} \mathrm{SOCH})\end{array}$ \\
\hline $5 f$ & $\mathrm{C}_{23} \mathrm{H}_{15} \mathrm{~N}_{2} \mathrm{O}_{3} \mathrm{~S}_{2}$ & 431 & 50 & $188-9$ & 64.0 & 66.4 & 3.48 & 3.4 & 6.40 & 6.3 & 14.8 & 14.7 & $\begin{array}{l}\text { 6.2-7.8 }(11 \mathrm{H} \mathrm{mtd} \text { aromatic } \\
\mathrm{C}_{4} \mathrm{H}, 4.6\left(1 \mathrm{H} \mathrm{d} . \mathrm{C}_{3} \mathrm{H}\right) \\
3.4(3 \mathrm{H} \mathrm{S} \mathrm{OH}, 2, \mathrm{~S}, 2 \mathrm{H} \text { of } \\
\left.\mathrm{CH}_{2}\right)\end{array}$ \\
\hline $5 \mathrm{~g}$ & $\mathrm{C}_{23} \mathrm{H}_{17} \mathrm{~N}_{2} \mathrm{O}_{3} \mathrm{~S}_{2}$ & 433 & 50 & $170-1$ & 63.7 & 63.01 & 3.92 & 3.8 & 6.46 & 6.3 & 14.7 & 14.6 & $\begin{array}{l}8.2-7.8(11 \mathrm{H} \text { mtd aromatic } \\
\mathrm{C}_{4} \mathrm{H}, 4.6\left(1 \mathrm{H} \mathrm{d} . \mathrm{C}_{3} \mathrm{H}\right), 125 \\
\mathrm{~S}, 1 \mathrm{H}, \mathrm{C}_{2} \mathrm{H}, 3.4(3 \mathrm{H} \mathrm{SOCH} \\
)\end{array}$ \\
\hline $5 \mathrm{~h}$ & $\mathrm{C}_{24} \mathrm{H}_{19} \mathrm{~N}_{2} \mathrm{O}_{3} \mathrm{~S}_{2}$ & 447 & 60 & $180-1$ & 64.4 & 64.3 & 4.25 & 4.2 & 6.26 & 6.2 & 14.3 & 14.2 & $\begin{array}{l}\text { 8.2-7.8 }(11 \mathrm{H} \mathrm{mtd} \text { aromatic } \\
\mathrm{C}_{4} \mathrm{H}, 4.6\left(1 \mathrm{H} \mathrm{d} . \mathrm{C}_{3} \mathrm{H}\right), 125 \\
\left.\mathrm{~S}, 1 \mathrm{H}, \mathrm{C}_{2} \mathrm{H}, 3.4(3 \mathrm{H} \mathrm{SOCH})_{3}\right)\end{array}$ \\
\hline
\end{tabular}


The antibacterial activity of both the series (4a-h) and (5a-h), respectively, have been carried out against some strain of bacteria. The results (Table 3 and 4) show that the prepared compounds are toxic against the bacteria. Compound $4 \mathrm{c}, 4 \mathrm{~d}, 4 \mathrm{~b}, 5 \mathrm{~b}, 5 \mathrm{~d}$ and $5 \mathrm{f}$ were found more active against the above microbes. The comparison of the antibacterial activity of these compounds with penicillin and sulphanilamide shows that these compounds have almost similar activity.

Table 3. Antibacterial Activity of compounds 4a-h

\begin{tabular}{ccccc}
\hline & \multicolumn{4}{c}{$\%$ age of zone of inhibition } \\
\cline { 2 - 5 } Compound & \multicolumn{2}{c}{ Gram +ve } & \multicolumn{2}{c}{ Gram-ve } \\
\cline { 2 - 5 } & $\begin{array}{c}\text { Bacillus } \\
\text { subtillis }\end{array}$ & $\begin{array}{c}\text { Staphyloccous } \\
\text { aureus }\end{array}$ & E.Coli & $\begin{array}{c}\text { Salmonella } \\
\text { typhi }\end{array}$ \\
\hline 4a & 58 & 67 & 44 & 68 \\
4b & 48 & 76 & 56 & 73 \\
4c & 75 & 85 & 80 & 65 \\
4d & 85 & 70 & 70 & 80 \\
$4 \mathrm{e}$ & 46 & 67 & 48 & 75 \\
4f & 70 & 63 & 65 & 50 \\
$4 \mathrm{~g}$ & 73 & 56 & 70 & 62 \\
$4 \mathrm{~h}$ & 83 & 73 & 80 & 75 \\
Penicillin & 85 & 65 & 75 & 75 \\
\hline
\end{tabular}

Table 4. Antibacterial Activity of compounds 5a-h

\begin{tabular}{ccccc}
\hline \multirow{2}{*}{ Compound } & \multicolumn{2}{c}{ Gram $+\mathrm{ve}$} & \multicolumn{2}{c}{ Gram -ve } \\
\cline { 2 - 5 } & $\begin{array}{c}\text { Bacillus } \\
\text { subtillis }\end{array}$ & $\begin{array}{c}\text { Staphyloccous } \\
\text { aureus }\end{array}$ & E.Coli & $\begin{array}{c}\text { Salmonella } \\
\text { typhi }\end{array}$ \\
\hline $5 \mathrm{a}$ & 60 & 72 & 60 & 60 \\
$5 \mathrm{~b}$ & 85 & 75 & 74 & 72 \\
$5 \mathrm{c}$ & 80 & 60 & 60 & 80 \\
$5 \mathrm{~d}$ & 73 & 80 & 80 & 70 \\
$5 \mathrm{e}$ & 70 & 80 & 85 & 83 \\
$5 \mathrm{f}$ & 70 & 75 & 80 & 76 \\
$5 \mathrm{~g}$ & 60 & 65 & 75 & 85 \\
$5 \mathrm{~h}$ & 75 & 65 & 78 & 70 \\
Sulphanilamide & 85 & 75 & 70 & 85 \\
\hline
\end{tabular}

\section{References}

1. Coppola K, PTC Int.Appl. 2001, 01108523.

2. Bian W, Jian Y N, Yang P, Xizng S H, Xuebzo S P and ZKexyeban , 23 ,231, C.A. 2001, 134, 237419.

3. Patil S and Bhagaval G, J. Int.Char. Soc. 1994, 71, 205.

4. Manian A K, Khadse G G and Sengupta S R, Indian drugs, 1993, 30, 324. 
5. Joshi M M, Bhagavat V S and Parvati J A, J. Ind. Chem. soc. 1995, 70, 647.

6. Stepanor D E, Evanov E I and Evanov RY, Russ. J. char. 2000, 70 (5), 784.

7. Sharma R C and Kumar D, J. Ind. Chem. Soc., 2000, 77,492.

8. Joshi H D, Sawale A R, Ingle R D, and Mane R A, Ind. J. Chem., 2000, 39, 967.

9. Ingle V S, Sawale A R, Ingle R D, and Mane R A, Ind. J. Chem., 2001, 40, 124.

10. Kagathara P, Upadhyay T, Doshi R and Parekh H H, Indian J. Heterocycl. Chem., $2000,10,9$.

11. Matsui N, Jpn. Kokai Tokkyo JP, 2000, 07, 652; Chem. Abstr., 2000, 132, 641094.

12. Desai K R, Asian J. Chem. Abstr, 2000, 132,279145.

13. Thaker K M, et. al., Ind. J. Chem., 2003, 42B, 1544.

14. Tripathy H and Mahaputra G N, J. Ind. Chem. Soc. 1975, 52,168.

15. (a) Barry A L, The antimicrobial susceptibility test: Principle and practices, $4^{\text {th }}$ ed. Philandelphia ,1976; Bio. Abstr. 1977, 64, 25183.

16. Kemp W, Organic Spectroscopy, $2^{\text {nd }}$ Edition. Basingtake, Macmillan, USA.

17. Furniss B, Hannaford A J, Smith P W G and Fatehell A R, Vage's Psnelial organic chemistry, Pearson Edition (Singapore) Pte.Ltd. Indian branch N.D, 2004. 


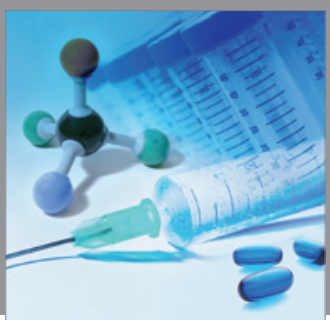

International Journal of

Medicinal Chemistry

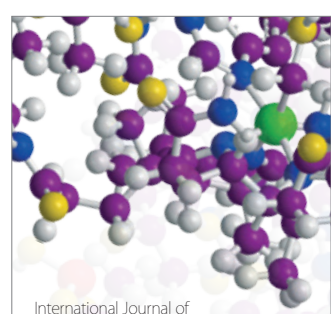

Carbohydrate Chemistry

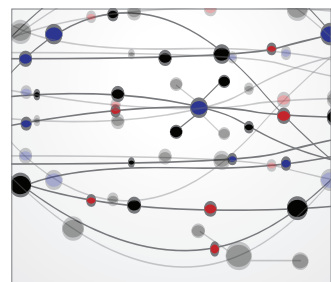

The Scientific World Journal
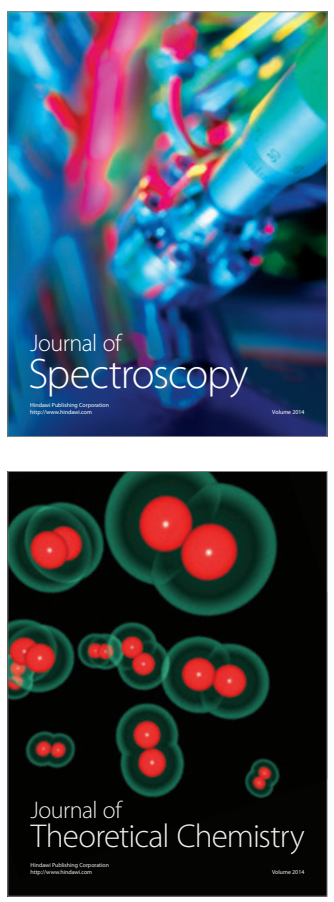
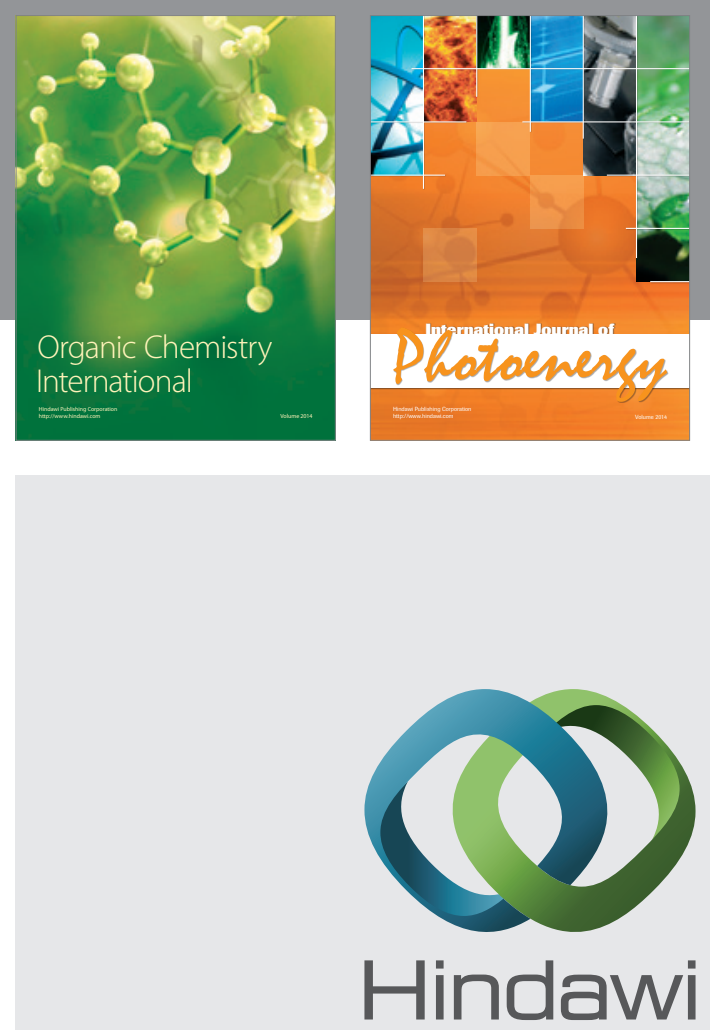

Submit your manuscripts at

http://www.hindawi.com
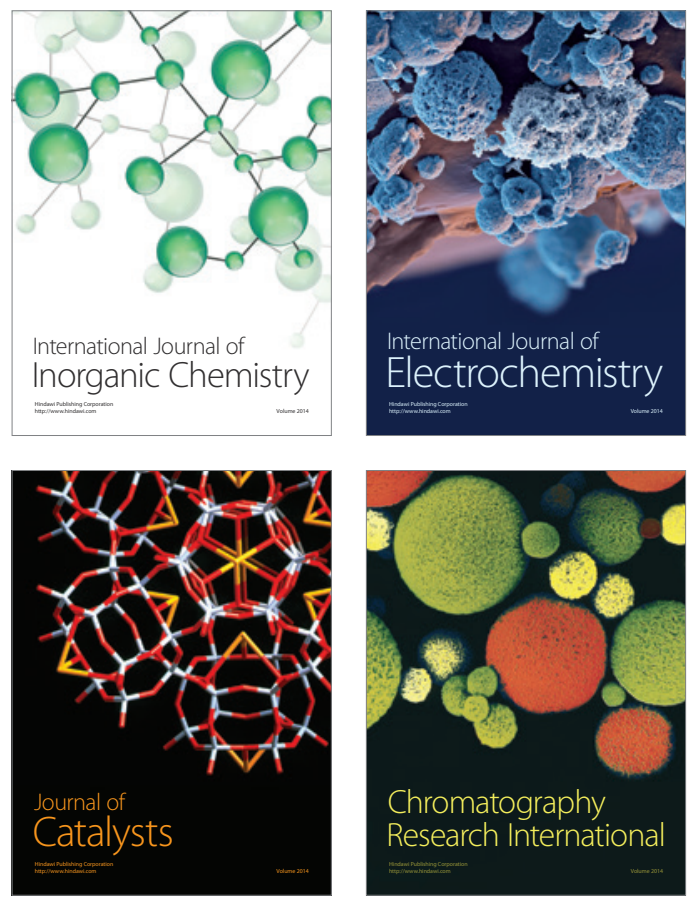
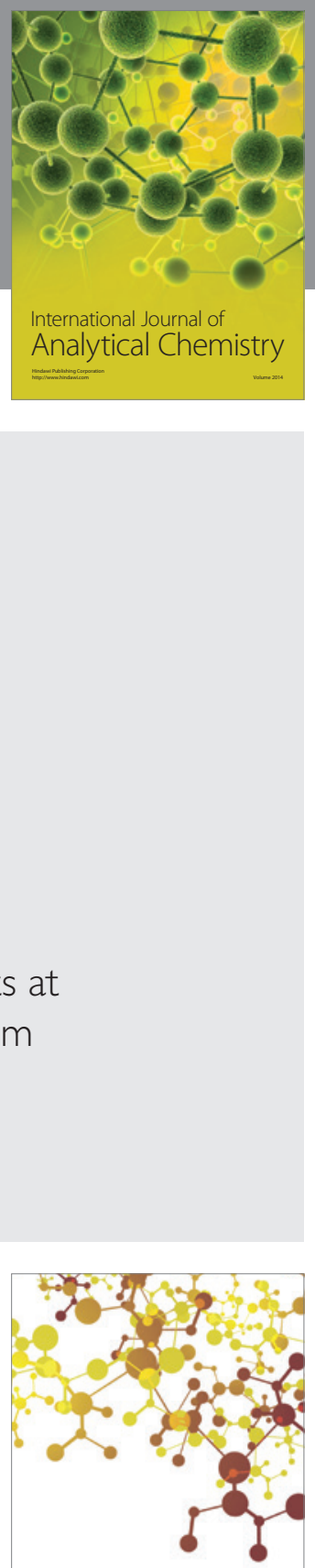

Journal of

Applied Chemistry
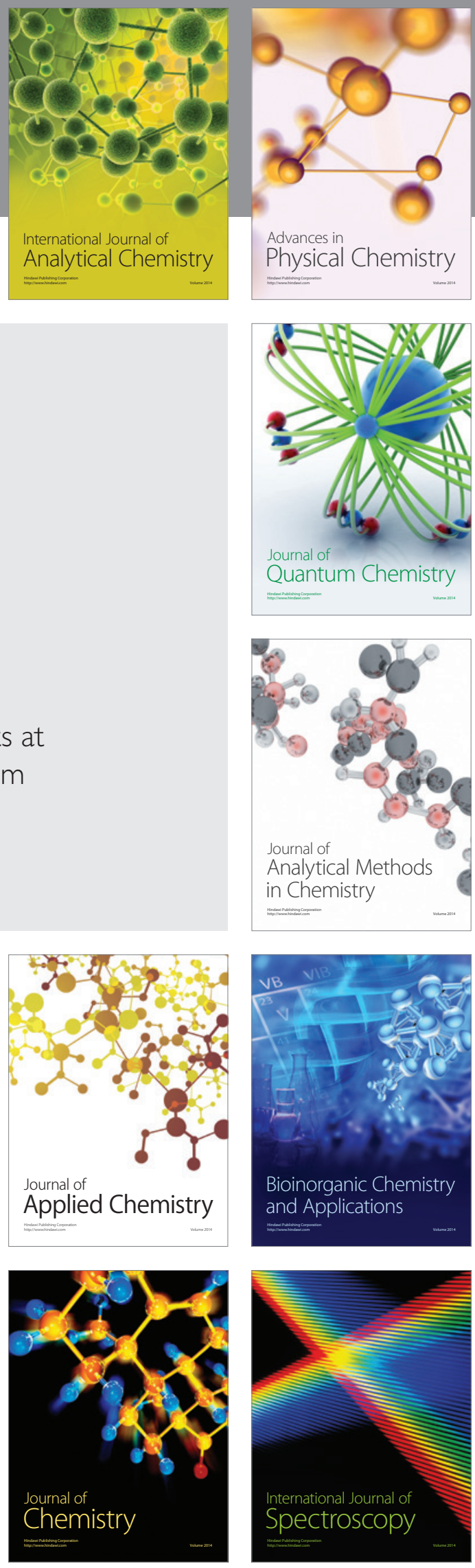